\title{
El Magdaleniense de Altamira
}

\author{
J. GonzAlez EChegaray
}

El yacimiento de la Cueva de Altamira, como se sabe, fue excavado ya por Sautuoia (1880), Alcalde del Río (1906) y Cartailhac y Breuil (1906), además de otros como E. Pedraja, J. Vilanova, Taylor-Ballota y E. Harlé, ofreciendo sendos niveles arqueológicos, atribuidos al Solutrense y Magdaleniense. La naturaleza de aquellas excavaciones y las colecciones de objetos publicadas y en algunos casos conservadas en los Museos de Santander y Altamira no permiten demasiadas precisiones acerca del tipo de Magdaleniense representado en el yacimiento de Altamira.

Obermaier, consciente del hecho, llevó a cabo dos campañas de excavación en el vestíbulo de Altamira entre 1924 y 1925, con el fin de precisar la estratigrafía y recoger nuevos datos sobre la naturaleza de ambos estratos. Sin embargo, tales excavaciones, realizadas en colaboración con el abate Breuil, el Conde de la Vega del Sella, el Conde de Bégouën y otros, no pudieron continuarse a causa de los desprendimientos producidos en la bóveda de este vestibulo, que, aparte de representar un serio peligro para la integridad de la cueva y de sus famosas pinturas, llegaron a constituir un serio riesgo para los excavadores, siendo herido el propio Obermaier en el curso de los trabajos, quien al parecer pudo salvar su vida de forma apurada.

Los resultados de la excavación de Obermaier fueron muy positivos, por lo que a la estratigrafía se refiere y francamente generosos en lo tocante al estudio de la fauna de ambos niveles y al mayor conocimiento de la industria del Solutrense, pero muy pobres por lo que al Magdaleniense se refiere (Breuil y Obermaier, 1935).

El estudio de las colecciones con obras de arte mueble fue retomado por Jordá (1964a y 1964b), Ripoll (1961), Barandiarán (1972) y Almagro (1976), y el de la industria solutrense por Soledad Corchón (1971). Por 
lo que a la industria del Magdaleniense se refiere, fuimos nosotros los que atendimos a su estudio (González Echegaray, 1960), intentando aplicar por vez primera los métodos estadísticos a su industria, así como a la del Solutrense (González Echegaray, 1971). Sin embargo, mientras que para el Solutrense manejamos una colección de 216 utensilios, para el Magdaleniense no pudimos contar más de 36 piezas, que con seguridad pertenecían a tal nivel, lo que evidentemente restaba valor estadístico a la prueba. Para obviar este problema, L. G. Straus (1974), que manejó los materiales en el Museo de Altamira y reestudió con éxito las colecciones solutrenses, y después de él, Pilar Utrilla (1981), decidieron atribuir al Magdaleniense varias cajas de material cuya etiquetación era deficiente, por lo que habían sido ya desechadas por nosotros previamente, todo ello en un afán, por otra parte plausible, de aumentar el valor cuantitativo de las colecciones.

Los resultados de esta operación no fueron muy afortunados, porque se habian atribuido al Magdaleniense materiales mezclados o revueltos, como claramente quedaría evidenciado con los resultados de la nueva excavación, según veremos más adelante. Los materiales solutrenses de Altamira, estudiados por Straus (1983) y por nosotros (1971) prácticamente no difieren desde el punto de vista estadístico, aunque Straus utiliza 522 útiles atribuidos con seguridad al Solutrense, y nosotros solamente 216. Una comparación entre los índices de ambos recuentos da cifras muy similares, especialmente por lo que se refiere a los epigrafes más representativos en esta colección, como el índice del raspador (IG), el del buril (IB), el del buril diedro tanto generalizado (IBd), como restringido $\left(\mathrm{IBd}^{\mathrm{r}}\right)$, y el índice del llamado Grupo Perigordiense (GP).

La comparación entre los índices de los recuentos para el Magdaleniense de Pilar Utrilla (1981) y los nuestros (1971) son algo más diferenciados, y esto no sólo porque la colección estudiada por Utrilla cuenta con 102 piezas, sino porque -como veremos después- en ella debe existir contaminación con elementos del Solutrense, al seguir la sugerencia de Straus, consistente en utilizar para el estudio cajas sin etiquetación garantizada, si bien esta prehistoriadora salva claramente su responsabilidad científica al decir: "A modo de hipótesis hemos intentado reunir los conjuntos cerrados de materiales sin nivel de Altamira, que no contuvieran ningún fósil conductor del Solutrense, añadiéndolos a los 36 objetos líticos bien datados» (Utrilla, 1981: 122).

Asi las cosas, y evidentemente no sólo por estas razones, sino por la importancia que tenía una revisión del yacimiento de Altamira, decidimos realizar una excavación en este yacimiento, lo que fue llevado a cabo, con el consiguiente permiso de la Dirección General de Bellas Ar- 


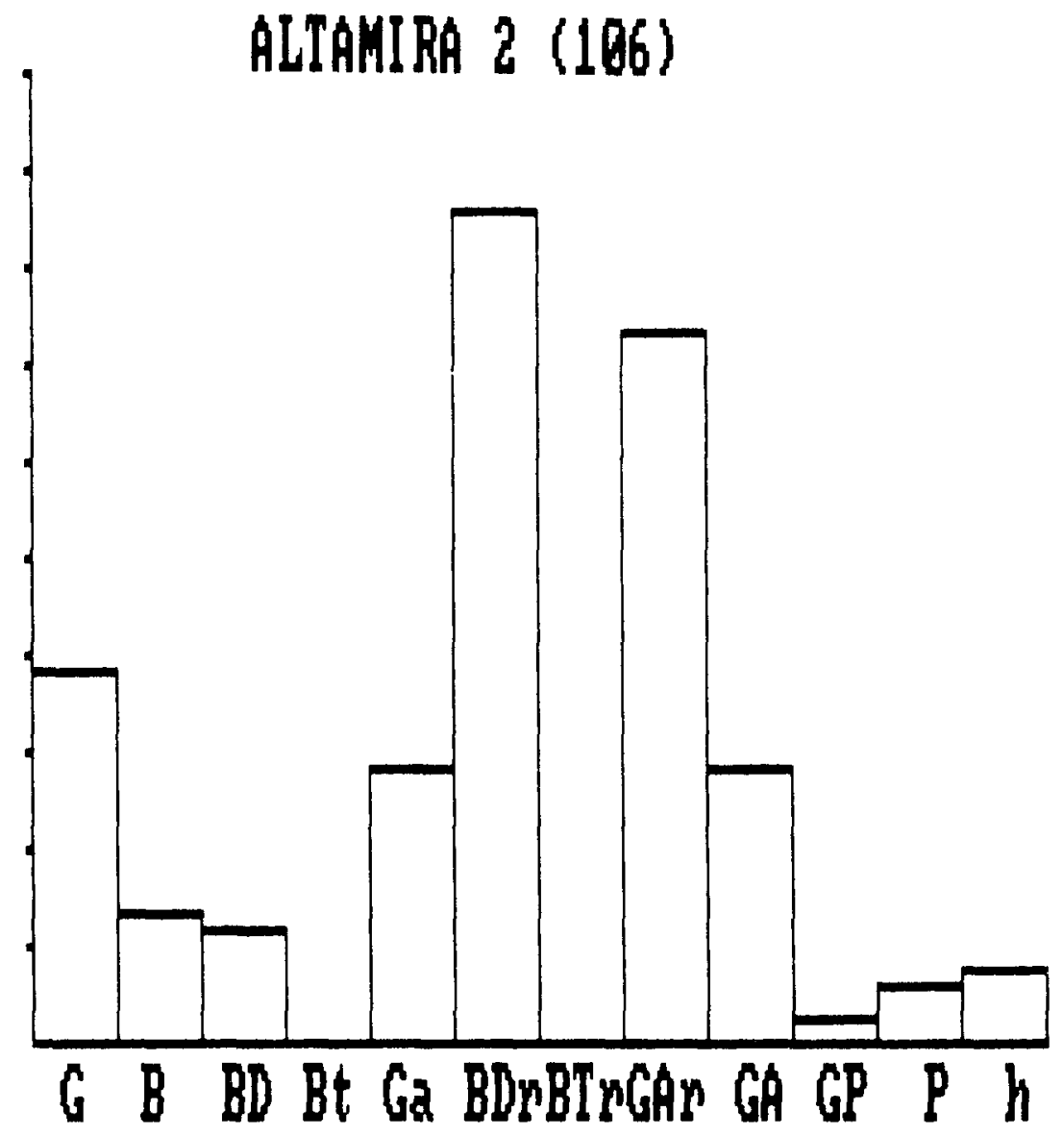

Fig. 1. Indices tipológicos del nivel 2. 
tes, en diciembre de 1981, siendo entonces el que esto escribe director del Centro de Investigación y Museo de Altamira, y habiendo solicitado el apoyo y colaboración de nuestros colegas L.G. Freeman, F. Bernaldo de Quirós y Victoria Cabrera, que tomaron parte en los trabajos, integrando un equipo. Desgraciadamente cuestiones de carácter político, dada la tensa situación existente entre aquella Dirección General y el Ayuntamiento de Santillana del Mar, aconsejaron suspender los trabajos, que no han podido reanudarse hasta hoy, pero el resultado de las investigaciones, aún en su mayoría inédito, fue muy positivo.

El Profesor Freeman tomó a su cargo el estudio del yacimiento con su estratigrafía y estructuras, el Dr. Bernaldo de Quirós el estudio de la materia prima de la industria lítica, así como las técnicas de talla, la Dra. Victoria Cabrera el estudio de la industria ósea y nosotros la tipología de los utensilios líticos. Aparte de esto, se hicieron análisis de $\mathrm{C}-14$ y se tomaron muestras para el del polen. El Profesor R. Klein ha realizado por su parte un estudio sobre la fauna. Esperamos que estas investigaciones puedan ver la luz en fecha próxima, aunque sería de desear que, una vez estabilizada la situación de Altamira, como de hecho ahora lo está, pudieran reanudarse los trabajos de excavación.

En cualquier caso vamos a dar a conocer ahora, por nuestra parte, el análisis tipológico de la nueva colección lítica del Magdaleniense de Altamira, obtenida en dichas excavaciones, publicando, si no la descripción detallada de las piezas, sí al menos los resultados estadísticos, con las listas tipológicas y los datos cuantitativos, así como gráficos e índices.

El total de piezas estudiadas asciende a 106, todas ellas de silex y pertenecientes al nivel, que en el curso de nuestra excavación recibió el nombre de Nivel 2, correspondiendo al mismo estrato que desde los tiempos de Sautuola se había venido teniendo por Magdaleniense. El análisis del C-14 dio la fecha de 15.910 230 B.P. (I-12012). Veinte años atrás nosotros mismos habiamos recogido muestras en el yacimiento para su análisis, que dieron la fecha prácticamente equivalente de $15.500 \pm 700$ B.P. (M-829). Ambas dataciones se solapan dado el amplio margen de error de la segunda, resultando naturalmente más afinada la primera, lo que nos habla de la existencia de un Magdaleniense Inferior Cantábrico en Altamira, que se desarrolla ligeramente después del año 14.000 a.C., hacia el 13.900 o 13.800 a.C.

A la vista de los adjuntos recuentos, índices y gráficos, hemos de hacer las siguientes consideraciones. En primer lugar, con relación a los anteriores estudios ya analizados, hemos de recalcar que el estudio de esta nueva colección tiende a ser en general más próximo a los resulta- 

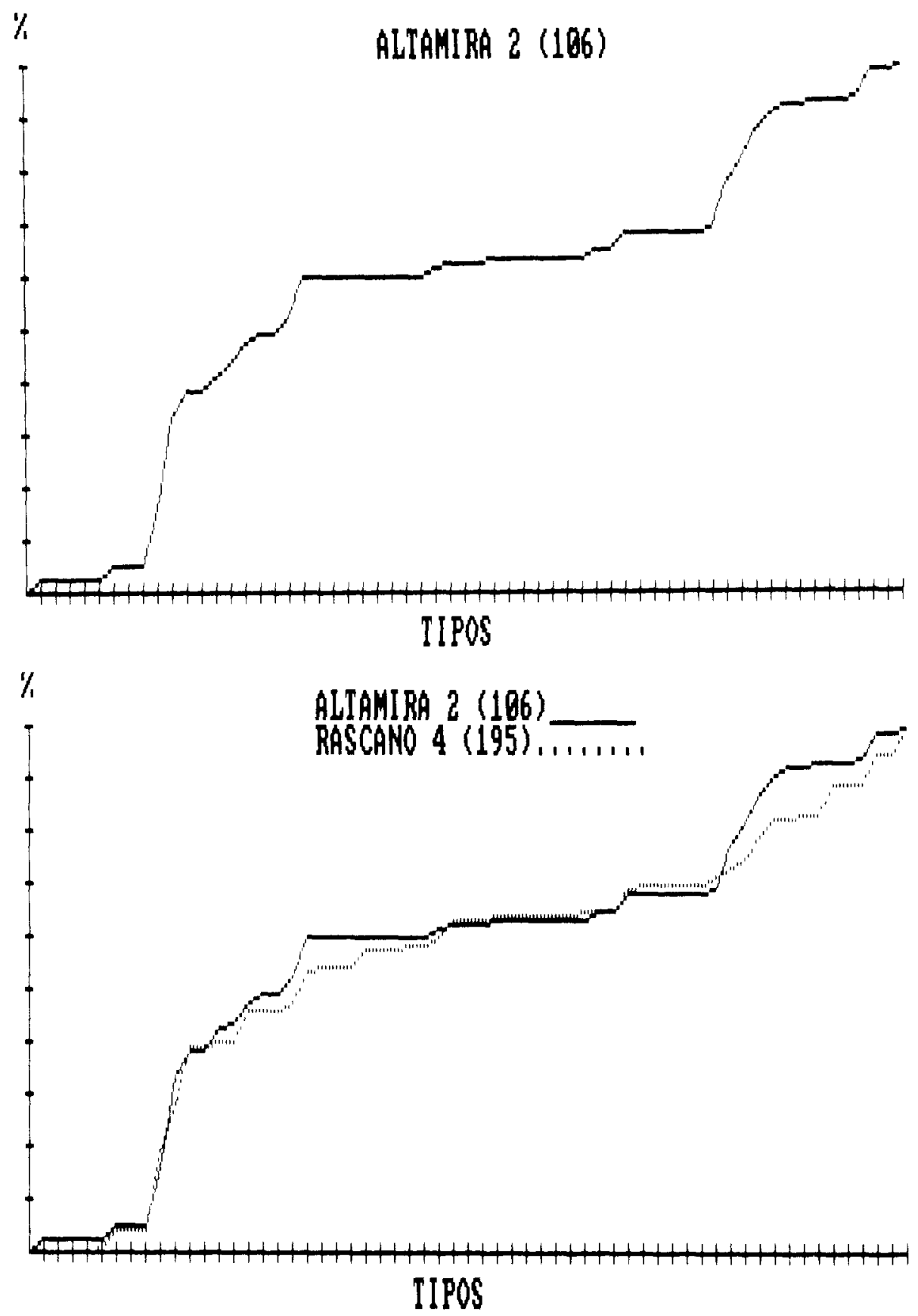

Fig. 2. Gráficas acumulativas. 
dos de nuestros recuentos de 1971, que a los de Straus-Utrilla, lo que se manifiesta claramente por la tendencia a subir el índice del raspador (IG), a descender el del buril (IB) y concretamente por las bajas cotas del buril diedro (IBd) y la inexistencia de buriles sobre truncatura (IBt). Por el contrario, el índice del raspador auriñaciense (IGA) tiende a subir claramente tanto en la colección estudiada por nosotros en 1971, como en la nueva de 1981. Igualmente el llamado Grupo Auriñaciense es alto en ambos recuentos, siendo en este aspecto aberrantes 0 indiferentes los índices del Grupo Perigordiense, los del perforador o los de las hojitas de dorso, lo que se explica por el escasísimo número de tales tipos en todas estas colecciones. Resulta, pues, que la nueva colección de Altamira confirma y aun refuerza las tendencias registradas en la pequeña colección estudiada por nosotros en 1971, en contra de los estudios más modernos que tendían a desdibujarlas. Por consiguiente ello permite desestimar el intento de añadir los materiales procedentes de cajas sin etiquetas. Más aún, la comparación de los índices IG, IBt e IGA hace sospechar que dichas cajas contenian materiales mezclados, procedentes del Solutrense, ya que lo que estos refuerzan son las tendencias claramente establecidas en colecciones de dicho nivel.

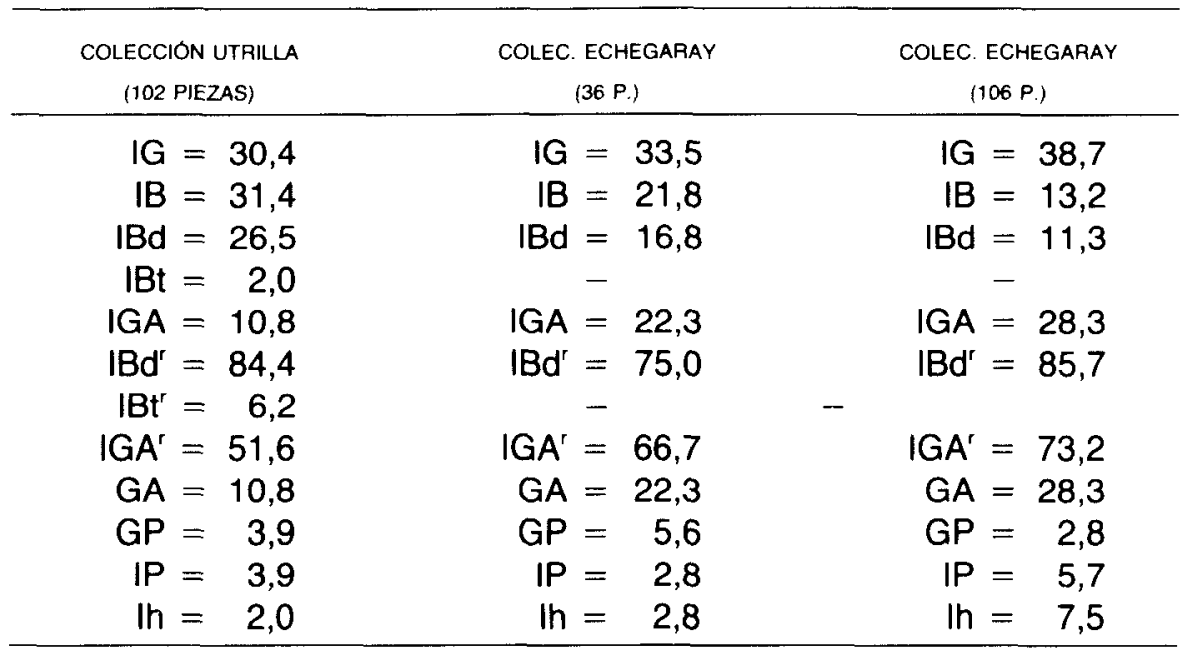

Algo que nos ha llamado siempre poderosamente la atención ha sido la escasez de hojitas de dorso en las viejas colecciones de Altamira, lo que no sólo afecta al Magdaleniense, sino incluso al Solutrense. Por contraste, otros yacimientos del Magadaleniense Inferior Cantábrico son muy ricos en hojitas retocadas de diversos tipos, especialmente la cueva del Juyo, en donde el porcentaje de hojitas en el nivel que da más, como 
es el 6 , asciende al 45,3 por 100 y en el que da menos, como es el nivel 4, no baja del 24,6 por 100 (González Echegaray, 1985). El Magadaleniense de Altamira según nuestros recuentos de 1971 daba sólo el 2,8 y según los de P. Utrilla aún menos: 2,0.

A primera vista podía pensarse que se tratara de un defecto en las técnicas de excavación, por falta del necesario cribado de las tierras, siendo excavaciones realizadas en 1924 y 1925. Sin embargo, esta suposición es absolutamente rechazable, puesto que los trabajos fueron dirigidos por Obermaier, que en su tiempo utilizaba el tamizado de las tierras *. Ahora nuestras excavaciones han venido a confirmar de manera evidente que la escasez de hojitas no se debe a un defecto técnico de la excavación, sino a una condición real del conjunto industrial representado por el yacimiento de Altamira. En efecto, a pesar de haberse practicado las técnicas más refinadas de excavación, el índice de las hojitas del Magdaleniense de Altamira no supera el 7,5. Existe pues, una verdadera facies dentro del Magadaleniense Inferior Cantábrico, entre cuyas características hay que señalar la pobreza de hojitas. Ya Pilar Utrilla había señalado con razón la existencia de dos facies: la del Juyo y la del País Vasco (Utrilla 1981: 295-296). Ahora parece ineludible añadir una tercera representada por Altamira (González Echegaray, 1985). A esta misma facies podría pertenecer el Magdaleniense Inferior de la Cueva del Castillo, niveles 8 y 7 , cuyo indice de hojitas es más bajo aún que en Altamira: 1,6 para el primero, y 1,1 para el segundo (Cabrera, 1984: 299 y 360-361).

Otro nivel que presenta muy pocas hojitas retocadas es el $4 b$ de Rascaño (González Echegaray y Barandiarán, 1981), con un índice de 0,8 . Sin embargo, el nivel 4 de esta cueva, con el que por otra parte está estrechamente emparentado hasta poder sospecharse que se trata de una simple variante topográfica dentro del yacimiento, arroja un índice del 12,8. En todo caso, si comparamos la curva acumulativa de Altamira y de Rascaño 4 se aprecia una gran similitud, que aboga, en efecto, por un parentesco estrecho. Ambos se caracterizan por índices muy similares en los raspadores: $I G=38,7$ para Altamira, y 39,5 para Rascaño y concretamente en el de los raspadores auriñacienses: IGA $=28,3$ para Altamira y 24,1 para Rascaño, y del llamado Grupo Auriñaciense: $G A=$ 28,3 para Altamira y 25,1 para Rascaño. Estas cifras adquieren más valor

* Algunas fotos obtenidas durante las excavaciones de Obermaier en Altamira permiten comprobar cómo se realizaba el cribado de las tierras. 
significativo al compararse con el resto, que permite apreciar unas proporciones igualmente equilibradas, en lineas generales.

Con Castillo beta, nivel 8 , pasa algo similar, ya que tiene un IG = 38,6 , un IB del orden de 17,5 (en Rascaño 4 es de 17,4), un IBd del orden de 8,9 (en Rascaño es de 8,2), y un IBt del orden de 3,6 (en Rascaño 4 es de 3,1 ). Estas cifras contrastan con el Juyo, donde el IG va de 28,8 como máximo en el nivel 4 , hasta 19,0 en el 4 sant., 16,0 en el nivel 7 , y 15,4 en el nivel 6 . El índice de buriles de este último yacimiento es más semejante al de Altamira, pero de nuevo el IGA vuelve a diverger claramente del grupo Altamira - Castillo - Rascaño 4, con cifras que van entre el 7,4 para el nivel 6 y el 19,6 para el nivel 4. Al GA le ocurre lo mismo, con valores que oscilan entre el 8,9 para el nivel 6, y el 19,8 para el 4. Parece, pues, que se confirma la existencia de un Magdaleniense tipo Altamira, rico en raspadores, con apreciable tradición auriñaciense y pobre en hojitas retocadas, frente a un Magdaleniense tipo El Juyo, más equilibrado por lo que a las cifras de raspadores y buriles se refiere, y muy rico en toda clase de hojitas retocadas, si bien de la serie de esta última cueva el nivel más próximo a la facies de Altamira es el nivel 4. En Rascaño el nivel 3 no pertenece a la facies de Altamira, sino que se aproxima más al Juyo, lo que no carece tampoco de un cierto valor cronológico, ya que, según las dataciones de $\mathrm{C}-14$, tal nivel es parcialmente contemporáneo de ese yacimiento, mientras que el 4 lo es de Altamira (ver cuadro sinóptico de dataciones en González Echegaray, 1985: 152). Por otra parte, conviene señalar también que en los niveles más profundos de la Cueva del Juyo, en concreto en el nivel 8 (del que existen ya clasificadas 597 piezas), cuya cronología tiende más a aproximarse a Altamira, se ven ya algunos sintomas que muestran una cierta tendencia hacia el modelo de este último yacimiento como una disminución del índice de hojitas retocadas, un aumento de los raspadores con un reforzamiento del tipo "auriñaciense", una práctica ausencia de los buriles de truncatura y un apreciable índice del GA. Tampoco se olvide que en este nivel empiezan ya a aparecer los omóplatos grabados (Freeman y González Echegaray, 1982), que caracterizan a los yacimientos de Altamira y El Castillo.

No quisieramos dar la impresión de que todo se reduce a un problema cronológico, porque esto seria, a nuestro juicio, deformar la realidad, pero tampoco queremos dejar de lado las implicaciones que lleva consigo una cronología bien establecida para los principales yacimientos de la región, en orden a comprender la estructura y composición de los conjuntos líticos del Magdaleniense cantábrico. 
El Magdaleniense de Altamira

ALTAMIRA 2

\begin{tabular}{|c|c|c|c|c|c|c|c|}
\hline TIPO & NUM & PORCEN & PORCEN CUM & IIPO & NUM & PORCEN & PORCEN CUM \\
\hline 1 & 2 & 0,0189 & 0,01887 & 55 & 0 & 0,0000 & 0,63208 \\
\hline 2 & 1 & 0,0094 & 0,02830 & 56 & 0 & 0,0000 & 0,63208 \\
\hline 3 & 0 & 0,0000 & 0,02830 & 57 & 0 & 0,0000 & 0,63208 \\
\hline 4 & 0 & 0,0000 & 0,02830 & 58 & 0 & 0,0000 & 0,63208 \\
\hline 5 & 0 & 0,0000 & 0,02830 & 59 & 0 & 0,0000 & 0,63208 \\
\hline 6 & 0 & 0,0000 & 0,02830 & 60 & 0 & 0,0000 & 0,63208 \\
\hline 7 & 0 & 0,0000 & 0,02830 & 61 & 1 & 0,0094 & 0,64151 \\
\hline 8 & 2 & 0,0189 & 0,04717 & 62 & 0 & 0,0000 & 0,64151 \\
\hline 9 & 0 & 0,0000 & 0,04717 & 63 & 1 & 0,0094 & 0,65094 \\
\hline 10 & 0 & 0,0000 & 0,04717 & 64 & 0 & 0,0000 & 0,65094 \\
\hline 11 & 2 & 0,0189 & 0,06604 & 65 & 3 & 0.0283 & 0,67925 \\
\hline 12 & 10 & 0,0943 & 0,16038 & 66 & 0 & 0,0000 & 0,67925 \\
\hline 13 & 16 & 0,1509 & 0,31132 & 67 & 0 & 0,0000 & 0,67925 \\
\hline 14 & 2 & 0,0189 & 0,33019 & 68 & 0 & 0,0000 & 0,67925 \\
\hline 15 & 6 & 0,0566 & 0,38679 & 69 & 0 & 0,0000 & 0,67925 \\
\hline 16 & 0 & 0,0000 & 0,38679 & 70 & 0 & 0,0000 & 0,67925 \\
\hline 17 & 4 & 0,0377 & 0,42453 & 71 & 0 & 0,0000 & 0,67925 \\
\hline 18 & 0 & 0,0000 & 0,42453 & 72 & 0 & 0,0000 & 0,67925 \\
\hline 19 & 0 & 0,0000 & 0,42453 & 73 & 1 & 0,0094 & 0,68868 \\
\hline 20 & 0 & 0,0000 & 0,42453 & 74 & 9 & 0,0849 & 0,77358 \\
\hline 21 & 1 & 0,0094 & 0,43396 & 75 & 5 & 0,0472 & 0,82075 \\
\hline 22 & 0 & 0,0000 & 0,43396 & 76 & 6 & 0,0566 & 0,87736 \\
\hline 23 & 0 & 0,0000 & 0,43396 & 77 & 3 & 0,0283 & 0,90566 \\
\hline 24 & 4 & 0,0377 & 0,47170 & 78 & 2 & 0,0189 & 0,92453 \\
\hline 25 & 2 & 0,0189 & 0,49057 & 79 & 0 & 0,0000 & 0,92453 \\
\hline 26 & 0 & 0,0000 & 0,49057 & 80 & 0 & 0,0000 & 0,92453 \\
\hline 27 & 3 & 0,0283 & 0,51887 & 81 & 0 & 0,0000 & 0,92453 \\
\hline 28 & 1 & 0,0094 & 0,52830 & 82 & 0 & 0,0000 & 0,92453 \\
\hline 29 & 4 & 0,0377 & 0,56604 & 83 & 0 & 0,0000 & 0,92453 \\
\hline 30 & 4 & 0,0377 & 0,60377 & 84 & 1 & 0,0094 & 0,93396 \\
\hline 31 & 0 & 0,0000 & 0,60377 & 85 & 0 & 0,0000 & 0,93396 \\
\hline 32 & 0 & 0,0000 & 0,60377 & 86 & 0 & 0,0000 & 0,93396 \\
\hline 33 & 0 & 0,0000 & 0,60377 & 87 & 0 & 0,0000 & 0,93396 \\
\hline 34 & 0 & 0,0000 & 0,60377 & 88 & 0 & 0,0000 & 0,93396 \\
\hline 35 & 0 & 0,0000 & 0,60377 & 89 & 1 & 0,0094 & 0,94340 \\
\hline 36 & 0 & 0,0000 & 0,60377 & 90 & 5 & 0,0472 & 0,99057 \\
\hline 37 & 0 & 0,0000 & 0,60377 & 91 & 0 & 0,0000 & 0,99057 \\
\hline 38 & 0 & 0,0000 & 0,60377 & 92 & 1 & 0,0094 & 1,00000 \\
\hline 39 & 0 & 0,0000 & 0,60377 & & & & \\
\hline 40 & 0 & 0,0000 & 0,60377 & & TOTAL & 106 & \\
\hline 41 & 0 & 0,0000 & 0,60377 & & TITAL & 100 & \\
\hline 42 & 0 & 0,0000 & 0,60377 & \multirow{2}{*}{\multicolumn{4}{|c|}{ INDICES }} \\
\hline 43 & 1 & 0,0094 & 0,61321 & & & & \\
\hline 44 & 1 & 0,0094 & 0,62264 & \multirow{2}{*}{\multicolumn{2}{|c|}{$\begin{array}{l}\text { IG } \\
\text { IB }\end{array}$}} & \multirow{2}{*}{\multicolumn{2}{|c|}{$\begin{array}{l}38,679 \\
13,208\end{array}$}} \\
\hline 45 & 0 & 0,0000 & 0,62264 & & & & \\
\hline 46 & 0 & 0,0000 & 0,62264 & \multicolumn{2}{|r|}{$\mathrm{IBd}$} & \multicolumn{2}{|c|}{$\begin{array}{l}13,208 \\
11,321\end{array}$} \\
\hline 47 & 0 & 0,0000 & 0,62264 & \multicolumn{2}{|r|}{ IBt } & \multicolumn{2}{|c|}{0,000} \\
\hline 48 & 0 & 0,0000 & 0,62264 & \multirow{2}{*}{\multicolumn{2}{|c|}{$\begin{array}{l}\text { IGA } \\
\text { IBdr }\end{array}$}} & \multicolumn{2}{|c|}{28,302} \\
\hline 49 & 0 & 0,0000 & 0,62264 & & & \multicolumn{2}{|c|}{85.714} \\
\hline 50 & 0 & 0,0000 & 0,62264 & & IBtr & \multicolumn{2}{|c|}{0,000} \\
\hline 51 & 1 & 0,0094 & 0,63208 & & IGAr & \multicolumn{2}{|c|}{73,171} \\
\hline 52 & 0 & 0,0000 & 0,63208 & & GA & \multicolumn{2}{|c|}{28,302} \\
\hline 53 & 0 & 0,0000 & 0,63208 & & GP & \multirow{2}{*}{\multicolumn{2}{|c|}{$\begin{array}{l}2,830 \\
5,660\end{array}$}} \\
\hline 54 & 0 & 0,0000 & 0,63208 & & IP & & \\
\hline 55 & 0 & 0.0000 & 0,63208 & & Ih & \multicolumn{2}{|c|}{7,547} \\
\hline
\end{tabular}




\section{BIBLIOGRAFIA}

AlCALde del Rio, H., 1906: Las pinturas y grabados de las cavernas prehistóricas de la provincia de Santander, Santander.

AlmaGro BASCH, M., 1976: "Los omoplatos decorados de la Cueva del Castillo", Trabajos de Prehistoria, 33: 9-99.

Barandiarán Maestu, I., 1972: Arte Mueble del Paleolítico Cantábrico, Zaragoza.

Breuil, H. y Obermaier, H., 1935: La Cueva de Altamira, Madrid, Tipografía de Archivos.

Cabrera Valdés, V., 1984: El yacimiento de la Cueva de "El Castillo" (Puente Viesgo, Santander), Bibl. Praehistórica Hispana, Vol. XXII, CSIC, Madrid.

Cartailhac, E. y Breuil, H., 1906: La Caverne d'Altamira à Santillane près Santander (Espagne). Monaco.

CORCHON, S., 1971: El Solutrense en Santander, ICC, Santander.

Freeman, L. G. y GonzAlez Echegaray, J., 1982: “Magdalenian mobile art from El Juyo (Cantabria)", Ars Praehistorica, 1: 161-167.

GonzÁlez Echegaray, J., 1960: “El Magdaleniense III de la Costa Cantábrica», BSAA, 26: 1-32.

- 1971: "Apreciaciones cuantitativas sobre el Magdaleniense III de la Costa Cantábrica», Munibe, 23: 323-327.

- 1985: "La Industria Lítica", en I. Barandiaran, L. G. Freeman et alii, Excavaciones en la Cueva del Juyo, CIMA, vol. 14, Madrid, págs. 121-153.

González Echegaray, J., Barandiaran, I. et alii, 1981: El Paleolítico Superior de la Cueva del Rascaño (Santander), CIMA, vol. 3, Santander.

JoRdÁ CerdÁ, F., 1964a: “Sobre técnicas, temas y etapas del Arte Paleolítico de la Región Cantábrica", Zephyrus XV: 5-25. 
- 1964b: «El Arte Rupestre Paleolítico de la Región Cantábrica. Nueva secuencia cronológico-cultural", en Prehistoric Art of the Western Mediterranean and the Sahara, Viking Fund Publications in Anthropology, N. ${ }^{\circ} 39$, págs. 47-81. 1964b

Ripoll Perello, E., 1961: "La cronología del Santuario de la Cueva de la Pileta y el Arte Rupestre", en Homenaje al Prof. Cayetano Mergelina, Murcia, págs. 1-13.

SANZ DE SAUTUOLA, M., 1880: Breves apuntes sobre algunos objetos prehistóricos de la provincia de Santander, Martínez, T., Santander.

Straus, L. G., 1975: “ ¿Solutrense o Magdaleniense Inferior Cantábrico? Significado de las diferencias», IDEA, 86: 781-791.

- 1977: "The Upper Palaeolithic cave site of Altamira (Santander, Spain)", Quaternaria, 19: 135-148.

- 1983: "El Solutrense Vasco-Cantábrico. Una nueva perspectiva", CIMA, vol. 10, Madrid.

Utrilla Miranda, P., 1981: “El Magdaleniense Inferior y Medio en la Costa Cantábrica", CIMA, vol. 4, Santander. 